OPEN ACCESS

Edited by:

Yu Lung Lau,

The University of Hong Kong,

Hong Kong

Reviewed by:

Lisa Bartnikas,

Boston Children's Hospital and Harvard Medical School, United States Qian Zhang,

The Rockefeller University, United States

*Correspondence:

Christopher J. A. Duncan christopher.duncan@ncl.ac.uk

Specialty section:

This article was submitted to Primary Immunodeficiencies,

a section of the journal

Frontiers in Immunology

Received: 31 October 2020 Accepted: 29 December 2020 Published: 18 February 2021

Citation:

Freil BJ, Hanrath AT, Chen R, Hambleton $S$ and Duncan CJA (2021) Life-Threatening Influenza, Hemophagocytic Lymphohistiocytosis and Probable Vaccine-Strain

Varicella in a Novel Case of Homozygous STAT2 Deficiency.

Front. Immunol. 11:624415. doi: 10.3389/fimmu.2020.624415

\section{Life-Threatening Influenza, Hemophagocytic Lymphohistiocytosis and Probable Vaccine-Strain Varicella in a Novel Case of Homozygous STAT2 Deficiency}

\author{
Bishara J. Freij ${ }^{1,2}$, Aidan T. Hanrath ${ }^{3}$, Rui Chen ${ }^{3}$, Sophie Hambleton ${ }^{3,4}$ \\ and Christopher J. A. Duncan ${ }^{3,5 *}$
}

\begin{abstract}
1 Pediatric Department, Beaumont Children's Hospital, Royal Oak, MI, United States, 2 Oakland University William Beaumont School of Medicine, Rochester, MI, United States, ${ }^{3}$ Immunity and Inflammation Theme, Translational and Clinical Research Institute, Newcastle upon Tyne, United Kingdom, ${ }^{4}$ Great North Children's Hospital, Newcastle upon Tyne Hospitals NHS Foundation Trust, Newcastle upon Tyne, United Kingdom, ${ }^{5}$ Royal Victoria Infirmary, Newcastle upon Tyne Hospitals NHS Foundation Trust, Newcastle upon Tyne, United Kingdom
\end{abstract}

STAT2 is a transcription factor that plays an essential role in antiviral immunity by mediating the activity of type I and III interferons (IFN-I and IFN-III). It also has a recently established function in the negative regulation of IFN-I signaling. Homozygous STAT2 deficiency is an ultra-rare inborn error of immunity which provides unique insight into the pathologic consequence of STAT2 dysfunction. We report here a novel genetic cause of homozygous STAT2 deficiency with several notable clinical features. The proband presented aged 12 months with hemophagocytic lymphohistiocytosis $(\mathrm{HLH})$ closely followed by clinical varicella, both occurring within three weeks of measles, mumps, and rubella (MMR) and varicella vaccinations. There was a history of life-threatening influenza A virus (IAV) disease 2 months previously. Genetic investigation uncovered homozygosity for a novel nonsense variant in STAT2 (c. 1999C>T, p. Arg667Ter) that abrogated STAT2 protein expression. Compatible with STAT2 deficiency, dermal fibroblasts from the child demonstrated a defect of interferon-stimulated gene expression and a failure to mount an antiviral state in response to treatment with IFN-I, a phenotype that was rescued by lentiviral complementation by wild type STAT2. This case significantly expands the phenotypic spectrum of STAT2 deficiency. The occurrence of life-threatening influenza, which has not previously been reported in this condition, adds STAT2 to the list of monogenetic causes of this phenotype and underscores the critical importance of IFN-I and IFN-III to influenza immunity. The development of probable vaccine-strain varicella is also a novel occurrence in STAT2 deficiency, implying a role for IFN-I/III immunity in control of attenuated varicella zoster virus in vivo and reinforcing the susceptibility to pathologic effects of live-attenuated viral vaccines in disorders of IFN-I 
immunity. Finally, the occurrence of HLH in this case reinforces emerging links to hyperinflammation in patients with STAT2 deficiency and other related defects of IFN-I signaling - highlighting an important avenue for further scientific enquiry.

Keywords: type I interferon, type I and type III interferon signaling, JAK-STAT signaling pathway, viral disease, hyperinflammation, interferon stimulated gene, inborn errors of antiviral immunity, varicella zoster virus

\section{INTRODUCTION}

Inborn errors of antiviral immunity are a rich source of insight into essential pathways of host defence against specific viral pathogens. In addition to teaching us fundamental but clinically relevant lessons, these ultra-rare disorders also inform the development of novel therapeutics and vaccines for viral disease. Discoveries in this field have shed light on the critical role played by innate type I and type III interferons (IFN-I and IFN-III) in human antiviral immunity [reviewed in $(1,2)]$. The importance of these pathways was highlighted recently by the discovery of defects of IFN-I immunity in patients with lifethreatening SARS-CoV-2 infection $(3,4)$.

IFN-I, comprising 13 subtypes of IFN $\alpha$, alongside IFN $\beta$, IFNE, IFNא and IFN $\omega$, was discovered more than 60 years ago as the mediator of 'viral interference' (5). The discovery of IFNIII (IFN $\lambda 1$-IFN $\lambda 4$ ) followed more recently (6-8). IFN-I signals through a single ubiquitously expressed receptor, a heterodimer of IFNAR1 and IFNAR2. By contrast, expression of the receptor for IFN-III-composed of IFNL1 and IL10RB-is more confined to mucosal surfaces and some immune cell subsets (9). Ligation of IFNAR or IFNLR activates an ostensibly similar downstream JAK-STAT signaling cascade culminating in the activation of two major transcription factor complexes-interferon stimulated gene factor 3 (ISGF3, composed of tyrosine phosphorylated STAT1, STAT2, and IRF9) and the gamma activated factor (GAF, homodimers of pSTAT1).

To date, genetic lesions have been identified in all major components of the canonical IFN-I and IFN-III signaling pathways (1). These disorders are recognized clinically by a phenotype of isolated vulnerability to severe and/or recurrent viral disease (with the exception of complete STAT1 deficiency where the pathogen susceptibility profile is broader, encompassing mycobacterial disease). STAT2 deficiency was among the first of these disorders to be discovered, and is characterized by susceptibility to both live-attenuated viral vaccines and naturally acquired viral pathogens (10-12), presumably reflecting the combined impact on systemic IFN-I and mucosal IFN-III responses.

STAT2 is a ubiquitously expressed transcription factor. Unlike other STAT family members, which are relatively promiscuous, STAT2 participates in a narrower range of cytokine signaling pathways (IFN-I and IFN-III only). It is frequently targeted by pathogenic viruses as a means of subverting IFN restriction (13). Recent findings indicate that STAT2 also plays an important role in immunoregulation, by supporting the function of USP18 (14) a key negative regulator of IFN-I signaling (15-18) — and also by sequestering STAT1 in the cytosol (19). Isolated failure of the regulatory function of STAT2 towards USP18 underlies a severe sterile autoinflammatory disease mediated by persistent, uncontrolled IFN-I signaling $(20,21)$.

Hyperinflammation is also increasingly recognized in a minority of STAT2-deficient patients (11), although the underlying pathomechanism is not known and consequently there is limited information about how to approach its management. Severe disease meeting clinical criteria for hemophagocytic lymphohistiocytosis (HLH) remains unusual in STAT2 deficiency, having been reported in only a single individual to date (12).

In addition to defining organizing principles of IFNs in antiviral immunity, monogenic disorders also provide information about key aspects of the host response to individual medically important viral pathogens, for example influenza (22). Susceptibility to life-threatening influenza in otherwise healthy individuals is an emerging clinical phenotype associated with several inborn errors of IFN immunity, including homozygous loss of function mutations in IRF7, IRF9, and TLR3 (23-25). Defects in STAT2 have not hitherto been associated with this phenotype.

In this report we describe a new case of complete STAT2 deficiency with several novel features, expanding the spectrum of disease and thereby informing understanding of the broader roles of IFN-I/III signaling in human immunity.

\section{MATERIALS AND METHODS}

\section{Ethics Statement and Consent}

This study was performed in accordance with the principles of the Helsinki declaration. Written parental consent was obtained for genetic testing. Ethical approval for studies on patient fibroblasts was granted by the NRES Committee North East Newcastle \& North Tyneside 1 (Ref: 16/NE/0002).

\section{Whole Exome Sequencing}

Whole exome sequencing of DNA extracted from whole blood of the proband was performed commercially on the Illumina platform by Invitae Corporation (California, USA). Alignment and variant analysis were undertaken according to current Genome Analysis Toolkit (GATK) joint calling best practice guidelines using the reference assembly GRCh37 (hg 19) for alignment. Carriage of the STAT2 variant by the proband, and all family members, was tested by Sanger sequencing of DNA isolated from whole blood by local diagnostic genetic laboratories according to standard methodologies. 


\section{In Silico Prediction Tools}

PhastCons is a method to determine the grade of conservation of a given nucleotide, given as a score from 0 to 1 (26). MutationTaster uses values which are precomputed and offered by UCSC (27). The Combined Annotation Dependent Depletion (CADD) score is a tool for integrating conservation and deleteriousness predictions (28).

\section{Cells, Cytokines, Immunoblotting}

Dermal fibroblasts from patient II:3 and three healthy controls were obtained by standard methods and cultured in Dulbecco's Modified Eagle's Medium supplemented by $10 \%$ foetal calf serum and $1 \%$ penicillin/streptomycin (DMEM-10). Human recombinant IFN $\alpha 2 \mathrm{~b}$ (Intron-A, Schering-Plough, USA) and IFN $\gamma$ (Immunikin, Boehringer Ingelheim, Germany) were used at $1000 \mathrm{IU} / \mathrm{ml}$. Immunoblotting was carried out as previously described (20). Antibodies used are described in Table 1.

\section{Viral Cytopathic Effect Reduction Bioassay}

Monolayers of dermal fibroblasts were infected with encephalomyocarditis virus (EMCV, kindly provided by R. E. Randall and D. Young, St. Andrew's University, UK) at $10^{5} \mathrm{pfu} /$ $\mathrm{ml}$ for $24 \mathrm{~h}$ before fixation with $4 \%$ formaldehyde and staining with $0.5 \%$ crystal violet. Plates were washed extensively then allowed to air dry. The remaining cell membrane-bound stain was solubilized with methanol and absorbance at $595 \mathrm{~nm}$ measured on a TECAN Sunrise plate reader (Tecan, Switzerland). Subsequent analysis was undertaken as previously described (20).

\section{Lentiviral Complementation}

The bicistronic lentivirus vector (pHR-SIN-CSGW) containing full-length WT human STAT2, or a control vector expressing GFP, was used (kindly provided by R.E. Randall, St Andrew's University, UK). Lentiviruses were produced by co-transfection of psPAX2, pCMV-VSV-G and lentiviral transfer plasmid in HEK293T cells using PEI (Sigma-Aldrich, Gillingham, UK) or FuGene (Promega, Wisconsin, USA). Virus containing supernatants were harvested at $48 \mathrm{~h}$ post-transfection, filtered $(0.45 \mu \mathrm{m}$ sterile filter) and concentrated 100 -fold with Lenti- $\mathrm{X}^{\mathrm{TM}}$ Concentrator (TaKaRa, Shiga, Japan) according to

TABLE 1 | Antibodies.

\begin{tabular}{llccc}
\hline Antibody & Host & Dilution & Source & Code \\
\hline STAT2 N-term & Mouse & $1: 2000$ & SCB & SC-1668 \\
STAT2 C-term & Rabbit & $1: 2000$ & SCB & SC-476 \\
pSTAT2 & Rabbit & $1: 2000$ & CST & 8841 \\
STAT1 & Rabbit & $1: 1000$ & CST & 9172 \\
pSTAT1 & Rabbit & $1: 1000$ & CST & 7649 \\
MX1/2/3 & Rabbit & $1: 1000$ & SCB & SC-50509 \\
IFIT1 (ISG56) & Goat & $1: 500$ & SCB & SC-82946 \\
RSAD2 & Rabbit & $1: 1000$ & CST & 13996 \\
$\alpha$-tubulin & Mouse & $1: 10,000$ & CST & 3873 \\
Anti-rabbit HRP-conjugated & Goat & Various & CST & 7074 \\
Anti-mouse HRP-conjugated & Horse & Various & CST & 7076 \\
Anti-goat HRP-conjugated & Rabbit & $1: 4000$ & Millipore & 401515 \\
\hline
\end{tabular}

CST, Cell Signaling Technologies; SCB, Santa-Cruz Biotechnology. manufacturer's instructions. Cells were spinoculated in six-well plates (1.5 h, $2000 \mathrm{rpm}$ ), with target or null control viral particles, at various dilutions in a total volume of $0.5 \mathrm{ml}$ DMEM-10 containing hexadimethrine bromide (Polybrene, $8 \mu \mathrm{g} / \mathrm{ml}$, Sigma-Aldrich). Cells were rested in virus-containing medium for $8 \mathrm{~h}$ then incubated in fresh DMEM-10 until $48 \mathrm{~h}$, when they were subjected to selection with $2.0 \mu \mathrm{g} / \mathrm{ml}$ puromycin (SigmaAldrich). Antibiotic-containing medium was refreshed every $72 \mathrm{~h}$.

\section{Statistical Analysis}

Continuous data from the EMCV CPE bioassay were normalized prior to parametric tests of significance (ANOVA with Sidak's post-test correction for multiple comparisons). Analysis was undertaken using GraphPad Prism version 8.0, with (corrected) two-tailed alpha $<0.05$ the threshold for significance.

\section{RESULTS}

\section{Case Summary}

We investigated a 12-month-old girl who presented with high fever, diarrhea, new onset vomiting, nasal congestion, cough, irritability, and lethargy. Six days before onset of this illness, she had been inoculated with age-appropriate vaccines (MMR, varicella zoster virus, hepatitis A, Haemophilus influenzae type $\mathrm{b}$, and the 13-valent pneumococcal conjugate vaccines) despite nasal congestion and clear rhinorrhea. The child was born to distantly related but healthy Honduran parents at 38 weeks of gestation. Her own medical history was remarkable for significant viral illness: at 9 months of age, she had a prolonged febrile illness with seizures, triggered by seasonal coronavirus HKU1 infection. At 10 months of age, she experienced severe influenza A pneumonia with very high fever and progressive respiratory failure despite treatment with oseltamivir, ceftriaxone, methylprednisolone and oxygen delivered by high-flow nasal cannulae. She required intubation and mechanical ventilation for 3 days but recovered.

On admission, her physical examination only showed nasal congestion. The laboratory parameters were largely unremarkable (Table 2). A partial septic screen yielded $>100,000$ colony forming units/ml of Escherichia coli from urine and a positive PCR for rhinovirus/enterovirus RNA from a nasopharyngeal swab sample. Blood cultures collected on days 4 and 6 and were sterile. High fever continued despite intravenous ceftriaxone for presumed pyelonephritis. She was on the Paediatric ward for 2 days with continuing high fever up to $41^{\circ} \mathrm{C}$. On day 3 , she was transferred to the Paediatric Intensive Care Unit (PICU) because of poor bilateral lower extremity perfusion that failed to improve on multiple fluid boluses. This was accompanied by persistent tachycardia, increased work of breathing, and a diffuse erythematous rash. Extensive imaging revealed no evidence of an infective focus in the renal tract (ultrasonography), chest (repeated plain x-radiography), abdomen/pelvis (contrast CT) or heart (echocardiography). Virology investigations were consistent with human herpes 
TABLE 2 | Laboratory data by day of fever.

\begin{tabular}{|c|c|c|c|c|c|c|c|c|c|c|}
\hline Laboratory data & d4 & d6 & d7 & d8 & d9 & d10 & d11 & d12 & d15 & Normal range \\
\hline Hemoglobin (g/dL) & 12.1 & 10.4 & 10.1 & 9.4 & 9.7 & 9.8 & 8.9 & 8.1 & 8.9 & $10.2-14.7$ \\
\hline Hematocrit (\%) & 34.4 & 30.1 & 29.2 & 26.9 & 27 & 26.3 & 25.7 & 24.5 & 27.3 & $30.8-43.7$ \\
\hline White blood cell count $\left(10^{9} / \mathrm{L}\right)$ & 7.6 & 3.5 & 2.3 & 1.4 & 1.5 & 2.7 & 3.5 & 5.6 & 9.1 & $6.0-17.5$ \\
\hline Absolute neutrophil count $\left(10^{9} / \mathrm{L}\right)$ & 4 & 2.2 & 1.2 & 0.8 & 0.8 & 1.1 & 1.4 & 2.4 & 3.1 & $1.5-8.5$ \\
\hline Absolute lymphocyte count $\left(10^{9} / \mathrm{L}\right)$ & 2.3 & 1 & 1 & 0.6 & 0.7 & 1.3 & 1.7 & 2.6 & 4.6 & $4.0-10.5$ \\
\hline Absolute monocyte count $\left(10^{9} / \mathrm{L}\right)$ & 1.2 & 0.2 & 0.1 & 9.1 & 0 & 0.2 & 0.3 & 0.4 & 0.7 & $0.3-0.9$ \\
\hline Platelet count $\left(10^{9} / \mathrm{L}\right)$ & 242 & 144 & 127 & 107 & 85 & 85 & 101 & 135 & 534 & $150-450$ \\
\hline AST (U/L) & 66 & 57 & & & 221 & 125 & 104 & 82 & 73 & $10.0-37.0$ \\
\hline ALT (U/L) & 57 & 38 & & & 100 & 94 & 85 & 78 & 55 & $5.0-30.0$ \\
\hline C-reactive protein (mg/L) & & 76 & & & & & & & & $0.0-8.0$ \\
\hline Ferritin (ng/ml) & & & & & 1980 & & & & & $12-207$ \\
\hline Lactate dehydrogenase (U/L) & & & & & 1043 & & & & & $190-420$ \\
\hline Triglycerides (mg/dL) & & & & & 289 & & & & & $30-120$ \\
\hline D-dimer (ng/ml) & & & & & & $>9,999$ & & & & 0-499 \\
\hline Fibrinogen (mg/dL) & & & & & & 71 & 65 & & & $175-375$ \\
\hline Soluble interleukin-2 receptor (pg/ml) & & & & & & 14740 & & & & $<1034$ \\
\hline
\end{tabular}

These abnormalities resolved on retesting in convalescence.

virus type 6 (HHV6) reactivation, based on positive blood PCR (23,500 (log 4.4) copies/ml of HHV-6 type B), positive HHV6 IgG at 1:80 (negative if $<1: 10)$ and negative $\operatorname{IgM}(<1: 20)$ on day 6 . Testing was not undertaken for MMR viruses; samples were not stored to enable retrospective testing. Persistent high fever was accompanied by worsening cytopenias, elevated triglycerides, elevated ferritin to $\geq 500 \mathrm{ng} / \mathrm{ml}$, and elevated soluble interleukin-2 receptor level (Table 2). These results met 5 of 8 criteria for the diagnosis of hemophagocytic lymphohistiocytosis (HLH) (13). An attempt at bone marrow aspiration on day 9 of admission was aborted due to apnea and profound desaturation. She was quickly resuscitated and returned to baseline within a few hours while hematologic parameters began spontaneously improving by day 10 . Fever abated after 15 days and the child was discharged on no antibiotics. Treatment with corticosteroids and/or other immunomodulators was planned after the bone marrow aspiration, but not administered because of clinical and laboratory evidence suggestive of spontaneous HLH resolution on day 10. The diagnosis was considered to be transient HLH triggered by HHV6 infection.

Immediately after hospital discharge she developed a diffuse vesicular rash from which varicella-zoster virus (VZV) DNA was detected by PCR. This occurred 20 days after receiving the varicella vaccine. She was treated with acyclovir for 5 days $(20$ $\mathrm{mg} / \mathrm{kg}$ every $6 \mathrm{~h}$ ) without complication. There was no attempt to ascertain by molecular testing whether VZV was the vaccine strain. However the child had no exposure to individuals with chickenpox or shingles at home or in hospital. Based on molecular typing of rash-associated virus in post-marketing varicella vaccine surveillance in the US and Europe, the timing of this rash is more consistent with vaccine-strain varicella (median onset 17-21 days post-vaccination) than with wild type varicella (median interval: $7-8$ days post-vaccination) $(29,30)$. Thus on a balance of probabilities, our patient likely experienced dissemination of vaccine-strain VZV rather than wild type varicella.

At 14 months of age, immunological work up was undertaken which revealed a polyclonal increase in IgG, protective antibody levels against measles, VZV, diphtheria, and tetanus and normal lymphocyte subset analysis (Table 3). Subsequently the proband has experienced multiple illnesses secondary to rhinovirus, parainfluenza virus type 3 , and human metapneumovirus (Table 4). Now 3 years of age, her physical growth and developmental milestones have been normal. Further live vaccines have been withheld. Prophylactic immunoglobulin therapy was not felt to be indicated.

\section{STAT2 Variant Identification}

Suspecting a genetic aetiology in this case, whole exome sequence analysis and addition/deletion testing of 207 genes associated with primary immunodeficiency was undertaken on whole blood DNA from the proband, identifying a homozygous predicted pathogenic variant in STAT2 (c.1999C $>$ T [p.Arg667Ter]) and a heterozygous variant in DOCK2 [c.54-1G>T], the latter of unlikely clinical relevance. Sanger sequencing analysis confirmed the presence of STAT2 c.1999C $>\mathrm{T}$ in the homozygous state in the

TABLE 3 | Immunophenotyping results, patient II:3.

\begin{tabular}{|c|c|c|}
\hline Parameter & Value & Normal range \\
\hline Total lymphocytes & 3,600 & $1600-6,700$ \\
\hline $\mathrm{CD} 3+\mathrm{CD} 4+$ & 1,008 & $1,000-4,600$ \\
\hline CD3+ CD8+ & 756 & $400-2,100$ \\
\hline CD3 & 1,368 & $600-2,700$ \\
\hline \multicolumn{3}{|l|}{ CD19+ } \\
\hline CD3- CD56+ & 216 & $200-1,200$ \\
\hline $\operatorname{lgG}(\mathrm{mg} / \mathrm{dL})$ & 1,170 & 300-900 \\
\hline $\operatorname{lgG1}(\mathrm{mg} / \mathrm{dL})$ & 979 & $160-562$ \\
\hline $\operatorname{lgG} 2$ (mg/dL) & 218 & $24-98$ \\
\hline $\operatorname{lgG} 3$ (mg/dL) & 90 & $17-64$ \\
\hline $\operatorname{lgG} 4$ (mg/dL) & 201 & $0-22$ \\
\hline $\operatorname{lgM}(\mathrm{mg} / \mathrm{dL})$ & 63 & $15-70$ \\
\hline $\operatorname{lgA}(\mathrm{mg} / \mathrm{dL})$ & 40 & $40-160$ \\
\hline $\operatorname{lgE}(\mathrm{IU} / \mathrm{ml})$ & 6 & $0.62-1.6$ \\
\hline Tetanus IgG (IU/ml) & 4.5 & $>0.1$ \\
\hline Diphtheria IgG (IU/ml) & 0.8 & $>0.1$ \\
\hline Measles IgG (AU/ml) & $>300$ & $\geq 30$ \\
\hline VZV IgG (Index units) & 1033 & $\geq 135$ \\
\hline
\end{tabular}


TABLE 4 | Summary of infection history in patient II:3.

\begin{tabular}{|c|c|}
\hline Age & Illness \\
\hline 9 months & $\begin{array}{l}\text { Prolonged fever with seizure; coronavirus HKU1 (non-COVID-19) } \\
\text { infection }\end{array}$ \\
\hline 10 months & Influenza $A(H 3)$ pneumonia, mechanical ventilation for 3 days \\
\hline $\begin{array}{l}12-13 \\
\text { months }\end{array}$ & $\begin{array}{l}\text { HLH with human herpes virus type } 6 \mathrm{~B} \text { replication, and varicella, } 20 \\
\text { days after Varivax, MMR, hepatitis A, Haemophilus influenzae type } \\
\text { b, and } 13 \text {-valent conjugate pneumococcal vaccines. Resolved } \\
\text { spontaneously. }\end{array}$ \\
\hline 14 months & $\begin{array}{l}\text { Pulmonary consolidation and fever for } 4 \text { days associated with } \\
\text { rhinovirus/enterovirus }\end{array}$ \\
\hline 15 months & $\begin{array}{l}\text { Rhinovirus/enterovirus upper respiratory tract infection with fever for } \\
7 \text { days }\end{array}$ \\
\hline 17 months & Suppurative otitis media with fever \\
\hline 24 months & Febrile parainfluenza virus type 3 upper respiratory tract infection \\
\hline 25 months & $\begin{array}{l}\text { Mixed upper respiratory tract infection with parainfluenza virus type } \\
\text { 3, human metapneumovirus, and rhinovirus/enterovirus }\end{array}$ \\
\hline 26 months & $\begin{array}{l}\text { Upper respiratory tract infection with rhinovirus/enterovirus, with } 5 \\
\text { days of fever up to } 39.3^{\circ} \mathrm{C} \text { and development of lower extremity } \\
\text { papular and urticarial rash }\end{array}$ \\
\hline
\end{tabular}

proband. This variant was heterozygous in both parents and a clinically unaffected sibling, consistent with segregation of an autosomal recessive trait (Figure 1A).

\section{The Arg667Ter Mutation Leads to Complete STAT2 Deficiency}

STAT2 c.1999C>T variant was absent from databases of genomic variation (gnomAD) (31). The variant introduced a premature stop codon in place of a conserved arginine residue at position 667 (p.Arg667Ter) in the SH2 domain of STAT2 protein (Figure 1B), which was predicted to be deleterious by in silico tools PhastCons (0.94, max 1.0), MutationTaster and CADD (36, max 36). To assess STAT2 protein expression, lysates were prepared from dermal fibroblasts obtained from the child. No truncated protein product was identified when probing primary dermal fibroblast lysates with antibodies raised against either N-terminal or C-terminal STAT2 antigens (Figure 2), indicative of complete STAT2 deficiency.

\section{Defective IFNAR But Preserved IFNGR Signaling}

STAT2 is activated by tyrosine phosphorylation downstream of the receptors for IFN-I and IFN-III, but not type II IFN (IFN $\gamma$ ), whereas STAT1 is activated by all IFN subtypes. We treated primary dermal fibroblasts from the child alongside healthy controls with recombinant IFN $\alpha 2 b$ or IFN $\gamma$. Probing whole cell lysates for tyrosine phosphorylated STAT2 and STAT1 proteins confirmed normal phosphorylation of STAT1 in response to IFN $\gamma$ in both patient and controls, implying that IFNGR signaling was intact in patient cells (Figure 2). STAT1 was also phosphorylated in patient lysates in response to IFN $\alpha 2 b$ at this early time point, although the abundance was reduced, as reported in STAT2-deficient cell-lines (19).

\section{Impaired ISG Expression and Induction of the Antiviral State}

The predicted consequence of STAT2 deficiency is to compromise the assembly of a functional ISGF3 complex, thus impairing the expression of interferon-stimulated genes (ISGs). Consistent with this prediction, the expression of several ISGF3dependent ISG proteins-MXA, IFIT1, and RSAD2 was absent in whole cell lysates prepared from STAT2-deficient patient fibroblasts exposed to IFN $\alpha 2$ b overnight (Figure 3A). To address the functional consequence of impaired ISG induction, we infected cells with encephalomyocarditis virus (EMCV), a picornavirus cytopathic for human cells in the absence of exogenous IFNs. In this experiment, fibroblasts were pretreated with IFN $\alpha 2 \mathrm{~b}$ overnight at a dose that was previously determined to prevent cytopathic effect (CPE) in control cells, then examined at $24 \mathrm{~h}$ post infection. STAT2-deficient cells were susceptible to CPE irrespective of IFN $\alpha 2 \mathrm{~b}$ exposure, confirming that induction of the antiviral state was compromised (Figure 3B).

\section{STAT2 Complementation Restored Functional IFN-I Responses}

To prove definitively that the loss of STAT2 protein was causal, we complemented patient cells with full-length human STAT2 delivered by lentiviral transduction (10). Complemented cells were compared to patient fibroblasts transduced in parallel with a control lentiviral vector expressing GFP. Lentiviral transduction of STAT2 in patient fibroblasts restored STAT2 protein expression and its phosphorylation in response to IFN $\alpha 2 \mathrm{~b}$ treatment (Figure $\mathbf{4 A}$ ). This translated into the restoration of both ISG induction and formation of a cellular antiviral state in response to IFN $\alpha 2 \mathrm{~b}$ (Figures 4B, C), thereby confirming the genotype-phenotype association. STAT2 overexpression led to the induction of MX1 and a partial antiviral state in the absence of IFN $\alpha 2 b$ exposure, as previously observed (10).
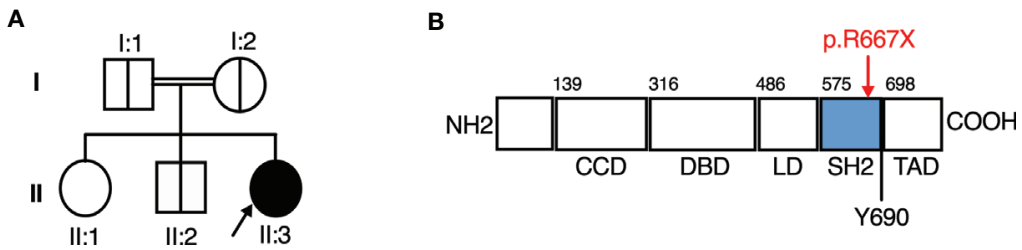

FIGURE 1 | A novel Arg677Ter variant causing autosomal recessive STAT2 deficiency. (A) Family pedigree. (B) STAT2 protein domains, showing the Arg677Ter variant. 


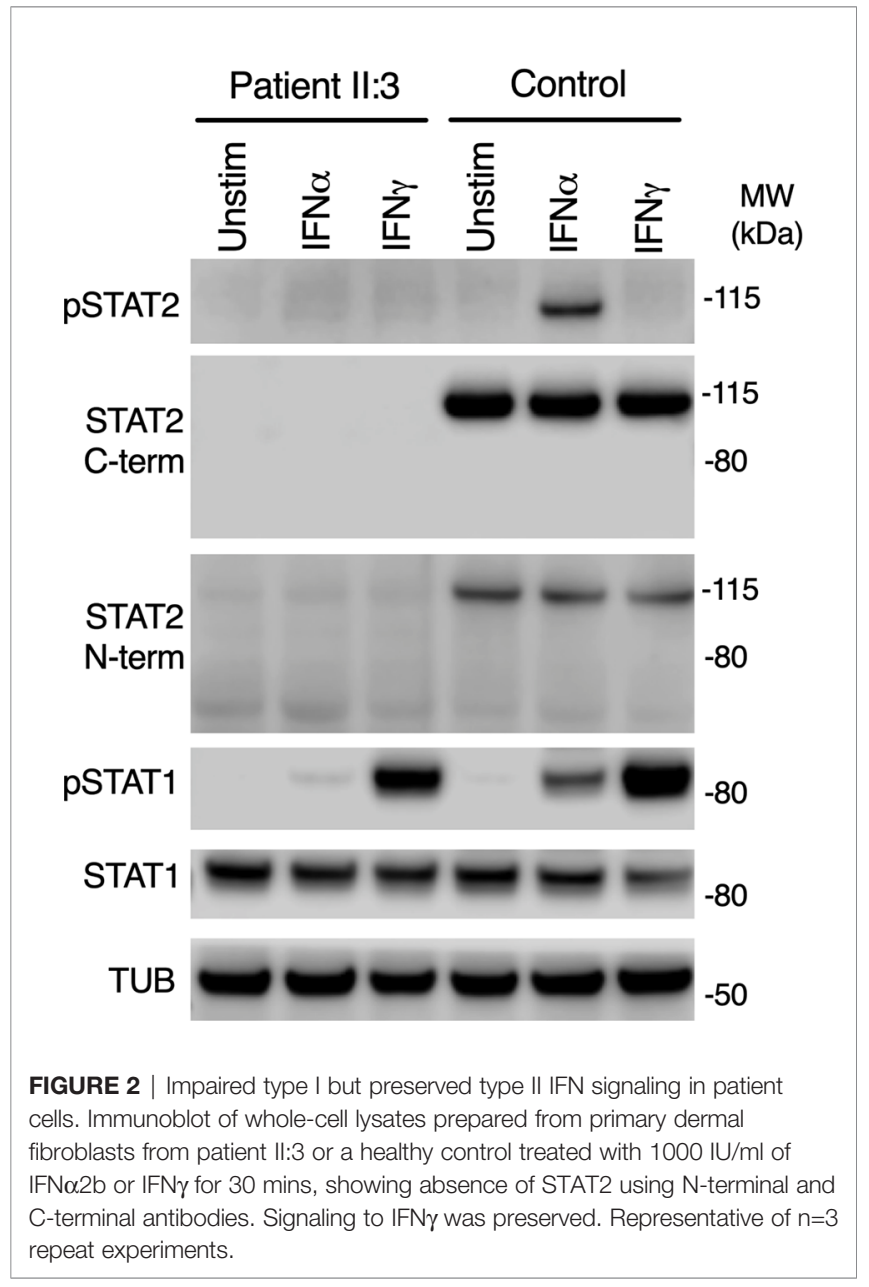

\section{DISCUSSION}

This case informs understanding of the function of STAT2 in both antiviral immunity and immunoregulation. STAT2 is unique among human STATs, by virtue of both its narrow spectrum of activity (within IFN-I/III pathways) and its direct participation in both positive and negative regulation. Defining the molecular and clinical consequences of STAT2 variants provides not only clinically relevant information, but also fundamental insight into the involvement of STAT2, and IFNI/III more generally, in human immunity.

Our data identify homozygous STAT2 deficiency as the fourth inborn error of IFN immunity underpinning severe influenza A virus (IAV) (23-25). Life-threatening IAV has been previously associated with inborn errors of IFN-I/III production due to homozygous loss of function defects in IRF7 (23) and more recently TLR3 (25) (previously recognized to underlie susceptibility to herpes encephalitis (32)). Whilst these disorders suggested that IFN-I/III signaling forms a critical aspect of the initial response to IAV infection, it was not possible to exclude the additional contribution of IFNindependent responses mediated by pattern-recognition receptor signaling. However, it is now clear that IFN-I/III signaling protects against severe manifestations of IAV - based on the recent report of life-threatening IAV in a patient with a homozygous loss of function variant in IRF9 (24), together with this report in STAT2 deficiency. It should be noted that severe IAV displays incomplete clinical penetrance in patients with mutated TLR3, IRF7, IRF9, or STAT2. This is not unexpected, since in the context of defects of pathogen defence there are multiple factors that likely contribute to variable expressivity, including: variable pathogen exposure, infecting dose, prior vaccination and compensation by adaptive immunity.

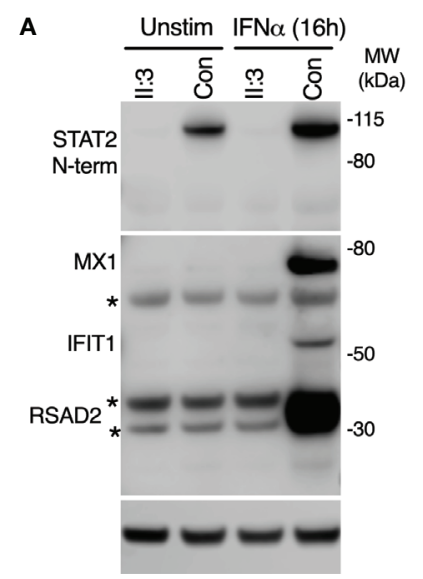

B

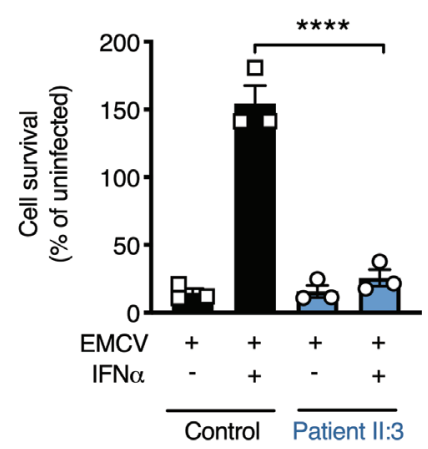

FIGURE 3 | Defective induction of the type I IFN dependent antiviral state. (A) Immunoblot analysis of whole cell lysates prepared from patient II:3 and control fibroblasts following overnight treatment with IFNo2b (1000 IU/ml) demonstrating the failure to induce the ISG products of MX1, RSAD2, and IFIT1 in patient fibroblasts. Stars represent non-specific bands. Representative of $n=3$ repeat experiments. (B) Encephalomyocarditis virus (EMCV) cytopathic effect reduction bioassay demonstrating the failure of IFN $2 \mathrm{~b}$ pre-treatment $(1000 \mathrm{IU} / \mathrm{ml})$ to induce the antiviral state in patient but not control fibroblasts. Displayed are mean $\pm \mathrm{SEM}$ of $n=3$ repeat experiments ${ }^{\star \star \star *} P<0.001$ ANOVA with Sidak's post-test. 
A

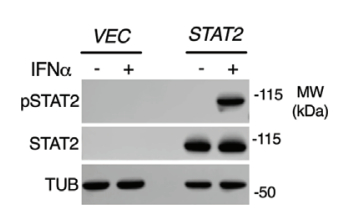

B

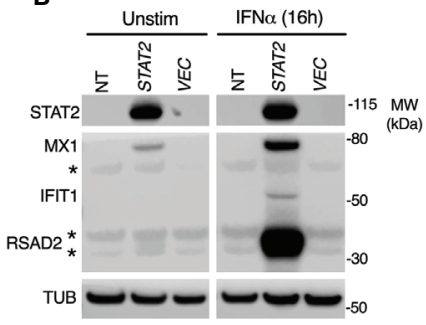

C

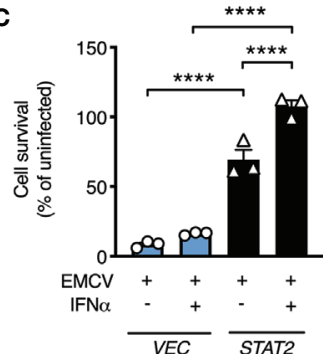

FIGURE 4 | STAT2 complementation rescues the defect of type I IFN signaling. Fibroblasts from patient II:3 were transduced by lentiviruses expressing either empty vector (GFP) or STAT2. Immunoblot analysis of whole cell lysates prepared from transduced fibroblasts demonstrating (A) restoration of STAT2 protein expression and tyrosine phosphorylation in response to $30 \mathrm{~min}$ IFNo2b $1000 \mathrm{IU} / \mathrm{ml}$ and (B) restoration of ISG expression in response to $16 \mathrm{~h}$ treatment with IFNo2b (1000 IU/ml) in STAT2 complemented patient fibroblasts. (C) EMCV cytopathic effect reduction bioassay demonstrating the failure of IFN $2 \mathrm{~b}$ pre-treatment (1000 IU/ml) to induce

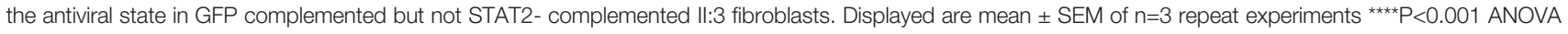
with Sidak's post-test.

All of the molecular defects hitherto associated with life threatening IAV impact the synthesis of, or transcriptional response to, both IFN-I and IFN-III. The relative contribution of IFN-I and IFN-III systems to IAV defence in humans remains an open question. A phenotype of vulnerability to severe IAV has not been reported to date in selective defects of either IFN-I signaling [i.e. IFNAR1 $(33,34)$ or IFNAR2 $(35)$ deficiency], nor IFN-III signaling [i.e. IL10RB deficiency (36)], suggesting that neither pathway in isolation is essential. Whilst this could be cautiously interpreted as evidence of functional redundancy, an important caveat is that only a handful of cases of IFNAR deficiency have been identified to date, and a broader phenotype might emerge as more cases are discovered and/or followed up. However this assessment has some support from studies of Ifnar $1^{-/-}$and Ifnlr ${ }^{-1-}$ mice, which suggest that IFN-I and IFN-III are effectively capable of functional compensation against severe disease due to influenza: only when both systems are simultaneously disabled, in Ifnar $1^{-1-}$ Ifnlr $1^{-/-}$double knockout mice, did mice consistently succumb to disease (37). However more recent data indicate that the IFN-III system plays a nonredundant role in protection of the upper airway, and in limiting transmission of IAV in mice (38), suggesting a distinct function for IFN-III. On this point it is also very relevant that specific defects of IFNAR signaling have recently been implicated in patients with life threatening SARS-CoV-2 infection, another pathogen naturally acquired via the respiratory tract (3). It is clear that SARS-CoV-2 has a broader cellular tropism and causes pathology in a wider range of tissues than IAV. Considering the mucosa-restricted expression of IFNLR compared to IFNAR, this broader tropism of SARS-CoV-2 might explain the apparently greater reliance of the host on IFN-I for protection. Nevertheless, specific mechanistic studies are required to determine the relative functional importance of IFN-I and IFN-III at the mucosa in human systems.

Disease caused by live-attenuated parenteral viral vaccines continues to serve as a clear signal of compromised IFNAR signaling $(10-12,34,35)$. Another novel observation in this case is the occurrence of clinical varicella following VZV immunization, which has not previously been described in
STAT2 deficiency. The implication is that vaccine-strain VZV may, like other live-attenuated vaccines such as MMR, be effectively controlled by innate IFNs in vivo (39), consistent with a previous report of VZV dissemination in a child with IRF9 deficiency (40). Regrettably in our case, molecular analysis was not undertaken at the time of varicella diagnosis to definitively prove the vaccine origin of VZV, nor was material stored to enable retrospective analysis. It is also notable that dissemination of MMR virus was not identified in this case, despite a suggestive temporal association between administration of MMR and the onset of clinical disease. Most individuals with molecular defects of IFN-I immunity that are known to have received MMR have developed pathologic dissemination. There are however exceptions. These include a patient with IFNAR1 deficiency who experienced no apparent illness following MMR, but nevertheless subsequently developed severe systemic disease due to dissemination of live-attenuated yellow fever vaccine (34). Another IFNAR1 deficient patient developed a self-limiting febrile illness following MMR, whereas her cousin died from complications of MMR (33). Thus, MMR dissemination is strongly associated with defects of IFN-I/III immunity, but is not inevitable. Again, it should be noted that in our case, blood and/or mucosal samples were not analyzed by PCR for MMR viral detection, and no clinical material was available to retrospectively test. Therefore occult MMR viral replication cannot be excluded as a potential contribution to HLH.

The occurrence of HLH is also unusual in STAT2 deficiency but underlines an emerging phenotype of immune dysregulation. Of the nine STAT2-deficient patients reported to date, three developed overt inflammatory manifestations, including prolonged febrile illness responsive to immunoglobulin therapy in two (11) and illness meeting HLH criteria in one (12). The pathomechanism accounting for hyperinflammation in STAT2 deficiency remains uncertain and represents an important avenue for future research. Interestingly, inflammatory disease has also been reported in other molecular defects of IFN-I/IFN-III signaling, including IFNAR2, STAT1 and IRF9 deficiency $(12,35,40,41)$. In most cases, hyperinflammation arose in the context of intercurrent viral infection, suggesting that viral infection might act as a trigger. 
In this regard, there was circumstantial evidence of reactivation of HHV6 in some of these cases $(35,41)$, as here, but whether this is causal or not remains unclear. However, other factors might also contribute to perpetuating and amplifying the dysregulated inflammatory response, including: (i) delayed control of viral replication (and/or herpesvirus reactivation) leading to a greater antigenic load; (ii) overactivity of compensatory immune pathways, such as IFN $\gamma$ or IL1 $\beta(42,43)$, and/or (iii) failure of STAT2dependent negative feedback upon type I IFN signaling. In the latter case, it is now well established that STAT2 plays an essential role in such regulation by supporting the regulatory function of USP18 (20, 21), and also by restraining IFNAR-independent activity of STAT1 (19). The concurrent loss of the regulatory activity of STAT2 might be an important contributor to these manifestations. From a therapeutic perspective, it remains unclear how best to treat hyperinflammation in STAT2 deficiency. The capacity of IFN $\gamma$ to compensate for antiviral activity in vitro might suggest a potential therapeutic role in controlling viral replication, however this approach may come at a cost of enhancing hyperinflammation. There have been clinical reports of responsiveness to IVIG or steroids $(11,41)$, although in our case the hyperinflammation resolved without specific treatment. More work is needed to dissect the pathomechanism and inform therapeutic strategies.

In summary, this case significantly expands our clinical understanding of STAT2 deficiency. As the first report of lifethreatening IAV in STAT2 deficiency, we can now add STAT2 to the list of genes implicated in this phenotype - a list that increasingly emphasizes the importance of IFN-I/III (23-25). We also report the first instance of disseminated vaccine-strain VZV in STAT2-deficiency, suggesting a role for innate IFNs in control of live-attenuated VZV vaccines. Finally, as only the second report of HLH in a patient with STAT2-deficiency, this case strengthens the notion that STAT2-deficient patients are vulnerable to hyperinflammation, motivating further investigation of the underlying mechanism(s) and highlighting the enduring capacity of monogenic diseases to uncover novel areas for scientific exploration.

\section{REFERENCES}

1. Duncan CJA, Randall RE, Hambleton S. Genetic Lesions of Type I Interferon Signalling in Human Antiviral Immunity. Trends Genet (2020) :S0168-9525 (20):30231-6.

2. Moens L, Meyts I. Recent human genetic errors of innate immunity leading to increased susceptibility to infection. Curr Opin Immunol (2020) 62:79-90.

3. Zhang Q, Bastard P, Liu Z, Le Pen J, Moncada-Velez M, Chen J, et al. Inborn errors of type I IFN immunity in patients with life-threatening COVID-19. Science (2020) 370(6515):eabd4570.

4. Bastard P, Rosen LB, Zhang Q, Michailidis E, Hoffmann HH, Zhang Y, et al. Autoantibodies against type I IFNs in patients with life-threatening COVID19. Science (2020) 370(6515):eabd4585.

5. Isaacs A, Lindenmann J. Virus interference. I. The interferon. Proceedings of the Royal Society of London. Ser B Biol Sci (1957) 147:258-67.

6. Kotenko SV, Gallagher G, Baurin VV, Lewis-Antes A, Shen M, Shah NK, et al. IFN-lambdas mediate antiviral protection through a distinct class II cytokine receptor complex. Nat Immunol (2003) 4:69-77.

7. Sheppard P, Kindsvogel W, Xu W, Henderson K, Schlutsmeyer S, Whitmore TE, et al. IL-28, IL-29 and their class II cytokine receptor IL-28R. Nat Immunol (2003) 4:63-8.

\section{DATA AVAILABILITY STATEMENT}

The raw data supporting the conclusions of this article will be made available by the authors, without undue reservation.

\section{ETHICS STATEMENT}

The studies involving human participants were reviewed and approved by NRES Committee North East-Newcastle \& North Tyneside 1 (Ref: 16/NE/0002). Written informed consent to participate in this study was provided by the participants' legal guardian/next of kin.

\section{AUTHOR CONTRIBUTIONS}

Clinical care: BF. Conception: BF, SH, CD. Experimental work: AH, RC, CD. Data analysis: AH, RC, CD. Funding acquisition: SH, CD. Manuscript writing: BF, CD. Manuscript reviewing and editing: $\mathrm{BF}, \mathrm{AH}, \mathrm{RC}, \mathrm{SH}, \mathrm{CD}$. All authors contributed to the article and approved the submitted version.

\section{FUNDING}

SH and CD are funded by the Wellcome Trust (207556/Z/17/Z and $211153 / Z / 18 / Z$ respectively). AH and $C D$ received additional grant funding from the British Medical Association.

\section{ACKNOWLEDGMENTS}

We are grateful to our patient and her parents for supporting this research. We thank R. Randall and D. Young for the kind gift of reagents.

8. Prokunina-Olsson L, Muchmore B, Tang W, Pfeiffer RM, Park H, Dickensheets H, et al. A variant upstream of IFNL3 (IL28B) creating a new interferon gene IFNL4 is associated with impaired clearance of hepatitis C virus. Nat Genet (2013) 45:164-71.

9. Sommereyns C, Paul S, Staeheli P, Michiels T. IFN-lambda (IFN-lambda) is expressed in a tissue-dependent fashion and primarily acts on epithelial cells in vivo. PLoS Pathog (2008) 4:e1000017.

10. Hambleton S, Goodbourn S, Young DF, Dickinson P, Mohamad SM, Valappil $\mathrm{M}$, et al. STAT2 deficiency and susceptibility to viral illness in humans. Proc Natl Acad Sci USA (2013) 110:3053-8.

11. Moens L, Van Eyck L, Jochmans D, Mitera T, Frans G, Bossuyt X, et al. A novel kindred with inherited STAT2 deficiency and severe viral illness. J Allergy Clin Immunol (2017) 139:1995-97.e9.

12. Alosaimi MF, Maciag MC, Platt CD, Geha RS, Chou J, Bartnikas LM. A novel variant in STAT2 presenting with hemophagocytic lymphohistiocytosis. J Allergy Clin Immunol (2019) 144:611-3.e3.

13. Henter JI, Horne A, Arico M, Egeler RM, Filipovich AH, Imashuku S, et al. HLH-2004: Diagnostic and therapeutic guidelines for hemophagocytic lymphohistiocytosis. Pediatr Blood Cancer (2007) 48:124-31.

14. Arimoto KI, Lochte S, Stoner SA, Burkart C, Zhang Y, Miyauchi S, et al. STAT2 is an essential adaptor in USP18-mediated suppression of type I interferon signaling. Nat Struct Mol Biol (2017) 24:279-89. 
15. Malakhov MP, Malakhova OA, Kim KI, Ritchie KJ, Zhang DE. UBP43 (USP18) specifically removes ISG15 from conjugated proteins. J Biol Chem (2002) 277:9976-81.

16. Sarasin-Filipowicz M, Wang X, Yan M, Duong FH, Poli V, Hilton DJ, et al. Alpha interferon induces long-lasting refractoriness of JAK-STAT signaling in the mouse liver through induction of USP18/UBP43. Mol Cell Biol (2009) 29:4841-51.

17. Francois-Newton V, Magno de Freitas Almeida G, Payelle-Brogard B, Monneron D, Pichard-Garcia L, Piehler J, et al. USP18-based negative feedback control is induced by type I and type III interferons and specifically inactivates interferon alpha response. PLoS One (2011) 6:e22200.

18. Malakhova OA, Kim KI, Luo JK, Zou W, Kumar KG, Fuchs SY, et al. UBP43 is a novel regulator of interferon signaling independent of its ISG15 isopeptidase activity. EMBO J (2006) 25:2358-67.

19. Ho J, Pelzel C, Begitt A, Mee M, Elsheikha HM, Scott DJ, et al. STAT2 Is a Pervasive Cytokine Regulator due to Its Inhibition of STAT1 in Multiple Signaling Pathways. PLoS Biol (2016) 14:e2000117.

20. Duncan CJA, Thompson BJ, Chen R, Rice GI, Gothe F, Young DF, et al. Severe type I interferonopathy and unrestrained interferon signaling due to a homozygous germline mutation in STAT2. Sci Immunol (2019) 4(42):eaav7501.

21. Gruber C, Martin-Fernandez M, Ailal F, Qiu X, Taft J, Altman J, et al. Homozygous STAT2 gain-of-function mutation by loss of USP18 activity in a patient with type I interferonopathy. J Exp Med (2020) 217(5):e20192319.

22. Ciancanelli MJ, Abel L, Zhang SY, Casanova JL. Host genetics of severe influenza: from mouse Mx1 to human IRF7. Curr Opin Immunol (2016) 38:109-20.

23. Ciancanelli MJ, Huang SX, Luthra P, Garner H, Itan Y, Volpi S, et al. Infectious disease. Life-threatening influenza and impaired interferon amplification in human IRF7 deficiency. Science (2015) 348:448-53.

24. Hernandez N, Melki I, Jing H, Habib T, Huang SSY, Danielson J, et al. Lifethreatening influenza pneumonitis in a child with inherited IRF9 deficiency. J Exp Med (2018) 215:2567-85.

25. Lim HK, Huang SXL, Chen J, Kerner G, Gilliaux O, Bastard P, et al. Severe influenza pneumonitis in children with inherited TLR3 deficiency. J Exp Med (2019) 216:2038-56.

26. Siepel A, Bejerano G, Pedersen JS, Hinrichs AS, Hou M, Rosenbloom K, et al. Evolutionarily conserved elements in vertebrate, insect, worm, and yeast genomes. Genome Res (2005) 15:1034-50.

27. Schwarz JM, Rodelsperger C, Schuelke M, Seelow D. MutationTaster evaluates disease-causing potential of sequence alterations. Nat Methods (2010) 7:575-6.

28. Rentzsch P, Witten D, Cooper GM, Shendure J, Kircher M. CADD: predicting the deleteriousness of variants throughout the human genome. Nucleic Acids Res (2019) 47:D886-94.

29. Sharrar RG, LaRussa P, Galea SA, Steinberg SP, Sweet AR, Keatley RM, et al. The postmarketing safety profile of varicella vaccine. Vaccine (2000) 19:916-23.

30. Goulleret N, Mauvisseau E, Essevaz-Roulet M, Quinlivan M, Breuer J. Safety profile of live varicella virus vaccine (Oka/Merck): five-year results of the European Varicella Zoster Virus Identification Program (EU VZVIP). Vaccine (2010) 28:5878-82.

31. Karczewski KJ, Francioli LC, Tiao G, Cummings BB, Alfoldi J, Wang Q, et al. The mutational constraint spectrum quantified from variation in 141,456 humans. Nature (2020) 581:434-43.
32. Zhang SY, Jouanguy E, Ugolini S, Smahi A, Elain G, Romero P, et al. TLR3 deficiency in patients with herpes simplex encephalitis. Science (2007) 317:1522-7.

33. Bastard P, Manry J, Chen J, Rosain J, Seeleuthner Y, AbuZaitun O, et al. Herpes simplex encephalitis in a patient with a distinctive form of inherited IFNAR1 deficiency. J Clin Invest (2021) 131(1):e139980.

34. Hernandez N, Bucciol G, Moens L, Le Pen J, Shahrooei M, Goudouris E, et al. Inherited IFNAR1 deficiency in otherwise healthy patients with adverse reaction to measles and yellow fever live vaccines. J Exp Med (2019) 216:2057-70.

35. Duncan CJ, Mohamad SM, Young DF, Skelton AJ, Leahy TR, Munday DC, et al. Human IFNAR2 deficiency: Lessons for antiviral immunity. Sci Transl Med (2015) 7:307ra154.

36. Glocker EO, Kotlarz D, Boztug K, Gertz EM, Schaffer AA, Noyan F, et al. Inflammatory bowel disease and mutations affecting the interleukin-10 receptor. N Engl J Med (2009) 361:2033-45.

37. Mordstein M, Neugebauer E, Ditt V, Jessen B, Rieger T, Falcone V, et al. Lambda interferon renders epithelial cells of the respiratory and gastrointestinal tracts resistant to viral infections. J Virol (2010) 84:5670-7.

38. Klinkhammer J, Schnepf D, Ye L, Schwaderlapp M, Gad HH, Hartmann R, et al. IFN-lambda prevents influenza virus spread from the upper airways to the lungs and limits virus transmission. Elife (2018) 7:e33354.

39. Duncan CJ, Hambleton S. Varicella zoster virus immunity: A primer. J Infect (2015) 71 Suppl 1:S47-53.

40. Bravo Garcia-Morato M, Calvo Apalategi A, Bravo-Gallego LY, Blazquez Moreno A, Simon-Fuentes M, Garmendia JV, et al. Impaired control of multiple viral infections in a family with complete IRF9 deficiency. J Allergy Clin Immunol (2019) 144:309-12.e10.

41. Burns C, Cheung A, Stark Z, Choo S, Downie L, White S, et al. A novel presentation of homozygous loss-of-function STAT-1 mutation in an infant with hyperinflammation-A case report and review of the literature. J Allergy Clin Immunol Pract (2016) 4:777-9.

42. Guarda G, Braun M, Staehli F, Tardivel A, Mattmann C, Forster I, et al. Type I interferon inhibits interleukin-1 production and inflammasome activation. Immunity (2011) 34:213-23.

43. Reboldi A, Dang EV, McDonald JG, Liang G, Russell DW, Cyster JG. Inflammation. 25-Hydroxycholesterol suppresses interleukin-1-driven inflammation downstream of type I interferon. Science (2014) 345:679-84.

Conflict of Interest: The authors declare that the research was conducted in the absence of any commercial or financial relationships that could be construed as a potential conflict of interest.

Copyright (C) 2021 Freij, Hanrath, Chen, Hambleton and Duncan. This is an openaccess article distributed under the terms of the Creative Commons Attribution License (CC BY). The use, distribution or reproduction in other forums is permitted, provided the original author(s) and the copyright owner(s) are credited and that the original publication in this journal is cited, in accordance with accepted academic practice. No use, distribution or reproduction is permitted which does not comply with these terms. 